\title{
Quantum groups under very strong axioms
}

by

\author{
Teo BANICA
}

\author{
Presented by Stanistaw L. WORONOWICZ
}

Summary. We study the intermediate quantum groups $H_{N} \subset G \subset U_{N}^{+}$. The basic examples are $H_{N}, K_{N}, O_{N}, U_{N}, H_{N}^{+}, K_{N}^{+}, O_{N}^{+}, U_{N}^{+}$, which form a cube. Any other example $G$ sits inside the cube, and by using standard operations, namely intersection $\cap$ and generation $\langle$,$\rangle , can be projected on the faces and edges. We prove that under the strongest$ possible axioms, namely (1) easiness, (2) uniformity, and (3) geometric coherence of the various projection operations, the eight basic solutions are the only ones.

Introduction. The unitary group $U_{N}$ has a free analogue $U_{N}^{+}$, constructed by Wang [27], and the study of the closed quantum subgroups $G \subset U_{N}^{+}$is a problem of general interest. These subgroups fit into the general theory developed by Woronowicz [29], [30], and can be studied by using representation theory methods. A generalization of Peter-Weyl theory is available for them, an analogue of the Tannakian duality holds as well, and the Haar functional can be computed via a Weingarten integration formula.

From the Tannakian perspective, the closed subgroups $G \subset U_{N}^{+}$containing the usual symmetric group, $S_{N} \subset G$, are the "simplest". Indeed, at the level of the associated Tannakian categories we have a reverse inclusion, $C_{G} \subset C_{S_{N}}$, and when coupling this with the well-known fact that $C_{S_{N}}$ is a very elementary object, namely the span of the category of set-theoretic partitions $P$, we are led in this way to pure combinatorics.

Thus, at the core of the classification work for compact quantum groups lies the question of classifying the intermediate subgroups $S_{N} \subset G \subset U_{N}^{+}$.

2010 Mathematics Subject Classification: Primary 46L65; Secondary 22E46.

Key words and phrases: quantum isometry, quantum reflection.

Received 28 January 2019.

Published online 12 April 2019. 
The work so far has focused on the "easy" case, where the Tannakian category of $G$ appears in the simplest possible way, namely $C_{G}=\operatorname{span}(D)$, for a certain subcategory $D \subset P$. See [11].

From the point of view of the Drinfeld-Jimbo twisting [18], [21], the easy quantum group theory is a $q=1$ theory. Twisting it at $q \in \mathbb{T}$ is not possible in general, because Woronowicz's formalism, based on $C^{*}$-algebras, requires $q \in \mathbb{R}$. Thus, the reasonable problem is that of twisting the theory over $\mathbb{T} \cap \mathbb{R}=\{ \pm 1\}$, and so at $q=-1$.

In Tannakian terms, the $q=-1$ twisting requires introducing a signature function in the implementation of the partitions $\pi \in P$ as linear maps. Such a signature function exists indeed, but is defined only on the subcategory $P_{\text {even }} \subset P$ of partitions having even blocks. Now since $\operatorname{span}\left(P_{\text {even }}\right)=C_{H_{N}}$, with $H_{N}$ being the hyperoctahedral group, the conclusion is that the $q=-1$ twisting procedure requires $H_{N} \subset G$.

Summarizing, when taking into account both the easiness philosophy and the Drinfeld--Jimbo twisting philosophy, we are led to the assumption $H_{N} \subset G \subset U_{N}^{+}$. See [2].

There are many examples of intermediate quantum groups $H_{N} \subset G \subset U_{N}^{+}$. Among them, there are eight basic solutions, which form a cube:

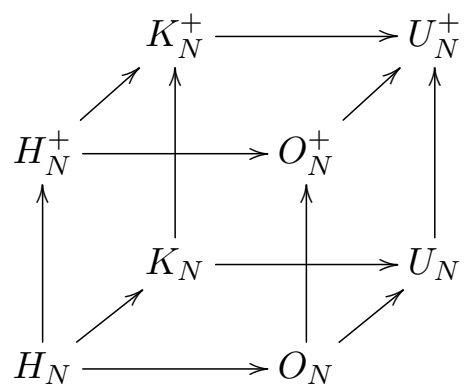

Here on the bottom face we have the orthogonal and unitary groups $O_{N}$ and $U_{N}$, and the hyperoctahedral group $H_{N}=S_{N} 2 \mathbb{Z}_{2}$ and its complex version $K_{N}=S_{N} 2 \mathbb{T}$. As for the quantum groups on the top face, these are liberations, from [5], 77, [27].

Quite remarkably, the above cube is an "intersection and generation" diagram. In order to explain this property, consider any of the six faces of the cube, say

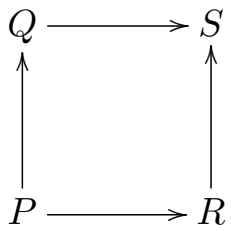


Given any such square diagram, we can use the intersection operation $\cap$ and the generation operation $\langle$,$\rangle , and impose the following conditions:$

$$
P=Q \cap R, \quad\langle Q, R\rangle=S .
$$

Now back to our cube, one can prove that this is indeed an intersection and generation diagram, as a consequence of various results in [5], [7], [11.

With this observation in hand, let us go back to the $H_{N} \subset G \subset U_{N}^{+}$ problem. Any solution $G$ sits inside the cube, and by using the operations $\cap$ and $\langle$,$\rangle , we can "project" this quantum group on various faces and edges$ of the cube. Under suitable assumptions, we end up with a "slicing" of the cube into eight small cubes.

To be more precise, we can first associate to $G$ six quantum groups:

$$
\begin{aligned}
G_{\text {class }} & =G \cap U_{N}, & G_{\text {free }} & =\left\langle G, H_{N}^{+}\right\rangle, \\
G_{\text {disc }} & =G \cap K_{N}^{+}, & G_{\text {cont }} & =\left\langle G, O_{N}\right\rangle, \\
G_{\text {real }} & =G \cap O_{N}^{+}, & & G_{\text {unit }}=\left\langle G, K_{N}\right\rangle .
\end{aligned}
$$

We then have inclusions between $G$ and these quantum groups:

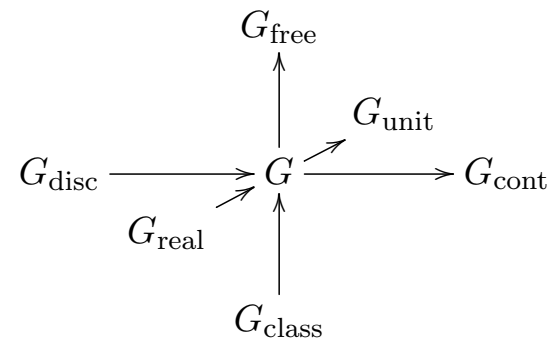

This diagram can be fit inside the original cube in the obvious way. Moreover, under suitable compatibility assumptions between the above operations, we can project on the edges as well, and we end up with a slicing of the original cube into eight small cubes.

Since we have a diagram formed by square subdiagrams, we can formulate:

Definition. We say that an intermediate subgroup $H_{N} \subset G \subset U_{N}^{+}$has the slicing property if the cube slicing that it produces is an intersection and generation diagram.

In order to complete our study, we will need one more concept. We recall that a family $G=\left(G_{N}\right)$ of quantum groups with $G_{N} \subset U_{N}^{+}$is called uniform when the following conditions are satisfied, with respect to the standard embeddings $U_{N-1}^{+} \subset U_{N}^{+}$:

$$
G_{N-1}=G_{N} \cap U_{N-1}^{+}
$$


This is something natural, algebrically speaking. At a more advanced level, this condition appeared in [11] in connection with the Bercovici-Pata bijection [13, and also in [1], [10] in connection with various noncommutative geometry questions.

With these preliminaries in hand, we can now formulate:

Theorem. Assume that $H_{N} \subset G \subset U_{N}^{+}$has the following properties:

- Easiness.

- Uniformity.

- Slicing property.

Then $G$ must be one of the basic eight quantum groups.

This will be our main result. Of course, it is quite philosophical. We will explain as well how to "build" on this result, by removing or modifying some of the axioms.

The paper is organized as follows: in $\S 1$ we recall the construction of the main cube, in $\S 2$ we explain in detail the above slicing procedure, in $\S 3$ we prove the above theorem, and in $\S 4$ we briefly discuss further classification results along these lines.

1. The cube. We use Woronowicz's quantum group formalism from [29], [30], under the extra assumption $S^{2}=$ id. To be more precise, the definition that we will need is:

Definition 1.1. Assume that $(A, u)$ is a pair consisting of a unital $C^{*}$ algebra $A$ and a unitary matrix $u \in M_{N}(A)$ whose coefficients generate $A$ such that the formulae

$$
\Delta\left(u_{i j}\right)=\sum_{k} u_{i k} \otimes u_{k j}, \quad \varepsilon\left(u_{i j}\right)=\delta_{i j}, \quad S\left(u_{i j}\right)=u_{j i}^{*}
$$

define morphisms of $C^{*}$-algebras $\Delta: A \rightarrow A \otimes A, \varepsilon: A \rightarrow \mathbb{C}, S: A \rightarrow A^{\text {opp }}$. We then write $A=C(G)$, and call $G$ a compact matrix quantum group.

The basic examples are the compact Lie groups $G \subset U_{N}$. Indeed, given such a group we can set $A=C(G)$, and let $u_{i j}: G \rightarrow \mathbb{C}$ be the standard coordinates, $u_{i j}(g)=g_{i j}$. The axioms are then satisfied, with $\Delta, \varepsilon, S$ being the functional-analytic transposes of the multiplication $m: G \times G \rightarrow G$, unit map $u:\{\cdot\} \rightarrow G$, and inverse map $i: G \rightarrow G$.

Another class of examples is provided by the abstract duals $G=\widehat{\Gamma}$ of the finitely generated discrete groups $\Gamma=\left\langle g_{1}, \ldots, g_{N}\right\rangle$. Indeed, we can set $A=C^{*}(\Gamma)$, and let $u=\operatorname{diag}\left(g_{1}, \ldots, g_{N}\right)$ be the diagonal matrix formed by the generators. Once again the axioms are satisfied, and when $\Gamma$ is abelian we have an identification $A=C(G)$. 
We are particularly interested here in the orthogonal group $O_{N}$, the unitary group $U_{N}$, the hyperoctahedral group $H_{N}=S_{N} \imath \mathbb{Z}_{2}$, and its complex version $K_{N}=S_{N}$ ¿ $\mathbb{T}$. These groups have free analogues, constructed in [5], [7], [27]:

Proposition 1.2. We have the following compact quantum groups, whose associated algebras are constructed by starting with an abstract $N \times N$ matrix $u=\left(u_{i j}\right)$ :

- $U_{N}^{+}$, obtained by imposing the conditions $u^{*}=u^{-1}, u^{t}=\bar{u}^{-1}$,

- $O_{N}^{+} \subset U_{N}^{+}$, obtained by further imposing the conditions $u=\bar{u}$,

- $K_{N}^{+} \subset U_{N}^{+}$, obtained via the conditions $u_{i j}^{*} u_{i j}=u_{i j} u_{i j}^{*}=p_{i j}=$ magic,

- $H_{N}^{+}$, obtained by imposing the conditions for both $O_{N}^{+}$and $K_{N}^{+}$,

with the "magic" condition stating that the $p_{i j}$ are projections with $\sum_{i} p_{i j}=$ $\sum_{j} p_{i j}=1$.

Proof. All this is standard, the idea in each case being that if $u=\left(u_{i j}\right)$ satisfies the conditions, then so do the matrices $u^{\Delta}=\left(\sum_{k} u_{i k} \otimes u_{k j}\right), u^{\varepsilon}=$ $\left(\delta_{i j}\right), u^{S}=\left(u_{j i}^{*}\right)$. Thus we can construct $\Delta, \varepsilon, S$ as in Definition 1.1 , by using the universal property of $C(G)$.

There are many things known about the above four quantum groups, in analogy with the known results about their classical counterparts. In the continuous case the passage $O_{N} \rightarrow U_{N}$ is best understood as a complexification at the Lie algebra level, and the free analogue of this fact states that $\mathrm{O}_{N}^{+} \rightarrow U_{N}^{+}$is a "free complexification", in a certain algebraic-geometry sense. In the discrete case, the key identifications $H_{N}=S_{N} \imath \mathbb{Z}_{2}$ and $K_{N}=S_{N} \imath \mathbb{T}$ have free counterparts $H_{N}^{+}=S_{N}^{+} \iota_{*} \mathbb{Z}_{2}$ and $K_{N}^{+}=S_{N}^{+} \iota_{*} \mathbb{T}$, with $S_{N}^{+}$being Wang's quantum permutation group [28], and with $2_{*}$ being Bichon's free wreath product operation [14]. For more on these topics, we refer to [5], [7].

In order to study these four quantum groups, and other quantum groups of the same type, we will need Woronowicz's Tannakian duality result from [30], in its "soft" form, worked out by Malacarne [22]. The precise statement that we need is as follows:

Proposition 1.3. The closed subgroups $G \subset U_{N}^{+}$are in correspondence with their Tannakian categories $C(k, l)=\operatorname{Hom}\left(u^{\otimes k}, u^{\otimes l}\right)$, the correspondence being given by

$$
C(G)=C\left(U_{N}^{+}\right) /\left\langle T \in \operatorname{Hom}\left(u^{\otimes k}, u^{\otimes l}\right) \mid k, l, T \in C(k, l)\right\rangle
$$

where all the exponents are by definition colored integers, with the corresponding tensor powers being defined by $u^{\otimes \emptyset}=1, u^{\otimes \circ}=u, u^{\otimes \bullet}=\bar{u}$ and multiplicativity.

Proof. As already mentioned, this result is taken from [22]. The idea is that we have a surjective arrow from left to right, and the injectivity can 
be checked by doing some algebra, and then by applying the bicommutant theorem as a main tool. See [22].

As a last ingredient, we will need the definition and basic properties of the $\cap$ and $\langle$,$\rangle operations for the closed subgroups of U_{N}^{+}$. We can proceed as follows:

Proposition 1.4. The closed subgroups of $U_{N}^{+}$are subject to $\cap$ and $\langle$, operations, constructed via the above Tannakian correspondence $G \mapsto C_{G}$, as follows:

(1) Intersection: defined via $C_{G \cap H}=\left\langle C_{G}, C_{H}\right\rangle$.

(2) Generation: defined via $C_{\langle G, H\rangle}=C_{G} \cap C_{H}$.

In the classical case, where $G, H \subset U_{N}$, we obtain in this way the usual notions.

Proof. Since the $\cap$ and $\langle$,$\rangle operations are clearly well-defined for the$ Tannakian categories, the operations in $(1,2)$ make sense indeed. As for the last assertion, it is something well-known, which follows from the definitions via an elementary computation.

The above statement is of course quite compact. It is possible to develop some more theory, with universality diagrams, other abstract aspects, and more examples as well. We refer to [15], where these operations are heavily used.

With these ingredients in hand, we can now go back to the basic four groups and four quantum groups, and formulate a key result about them:

THEOREM 1.5. The basic quantum unitary and quantum reflection groups, with the inclusions between them, form a cubic diagram:

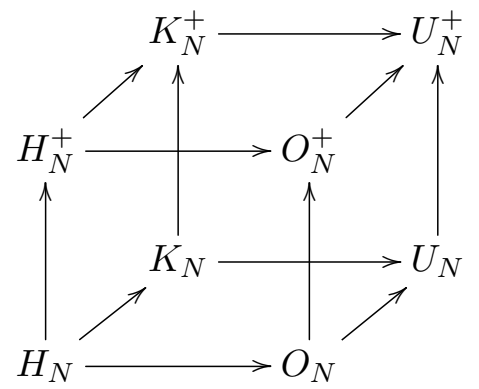

Moreover, this is an intersection/generation diagram, in the sense that for any of its square subdiagrams $P \subset Q, R \subset S$ we have $P=Q \cap R$ and $\langle Q, R\rangle=S$.

Proof. The first assertion is clear from the definitions. In order to prove the second assertion, we must compute the Tannakian categories of our eight quantum groups. 
For this purpose, we use the easy quantum group philosophy. Let us recall from [11] that associated to any partition $\pi \in P(k, l)$ between an upper row of $k$ points and a lower row of $l$ points is the following linear map between tensor powers of $\mathbb{C}^{N}$ :

$$
T_{\pi}\left(e_{i_{1}} \otimes \cdots \otimes e_{i_{k}}\right)=\sum_{j_{1}, \ldots, j_{l}} \delta_{\pi}\left(\begin{array}{ccc}
i_{1} & \ldots & i_{k} \\
j_{1} & \ldots & j_{l}
\end{array}\right) e_{j_{1}} \otimes \cdots \otimes e_{j_{l}} .
$$

Here $\delta_{\pi} \in\{0,1\}$ is a Kronecker type symbol, whose value depends on whether the indices fit or not, when put in the obvious way on the legs of the partition. See [11].

With this construction in hand, the result regarding our eight quantum groups, which is well-known from Brauer's paper [16] and from a number of extensions, including [5], 7], [11], is that we obtain categories of the following type:

$$
C(k, l)=\operatorname{span}\left(T_{\pi} \mid \pi \in D\right) .
$$

To be more precise, let $P_{\text {even }}$ be the set of partitions whose blocks all have even size, let $P_{2} \subset P_{\text {even }}$ be the set of pairings, let $\mathcal{P}_{\text {even }} \subset P_{\text {even }}$ be the set of partitions which are "matching" in the sense that \#० $=\# \bullet$ in each block, when counting the upper legs with the - sign and the lower legs with the + sign, and finally let $N C_{\text {even }} \subset P_{\text {even }}$ be the set of partitions which are noncrossing. We have so far four sets of partitions, and by further intersecting these sets we obtain four more sets, denoted $P_{2}, N C_{2}, \mathcal{N C}_{\text {even }}, \mathcal{N C}_{2}$.

Observe that these $4+4$ sets of partitions are "categories of partitions" in the sense of [11]: they are stable under vertical and horizontal concatenation of partitions, and under upside-down turning.

With these conventions, the above-mentioned result states that the quantum groups in the statement come from the following categories of partitions:

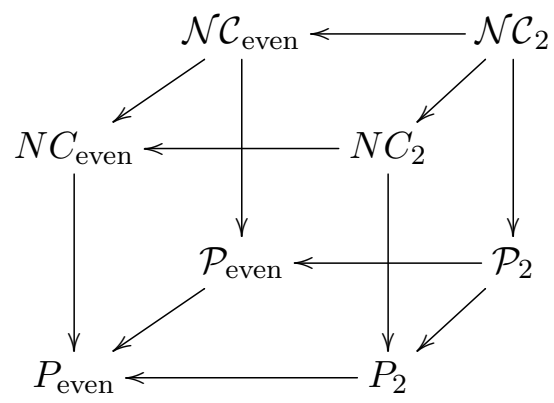

Now observe that this diagram of categories of partitions is an intersection and generation diagram, as is elementary to check.

Getting back to quantum groups, via the Tannakian duality operation, $D \rightarrow G_{D}$, it follows from Proposition 1.4 that we have the formulae

$$
G_{D \cap E}=\left\langle G_{D}, G_{E}\right\rangle, \quad G_{\langle D, E\rangle}=G_{D} \cap G_{E} .
$$


Thus, our intersection and generation diagram of categories of partitions gets transformed into an intersection and generation diagram of quantum groups, as stated.

We will heavily use the above result, as well as the various technical ingredients developed in its proof. In addition to what has been said, let us mention that the quantum groups whose Tannakian categories are of the form $C=\operatorname{span}(D)$, as in the above proof, are called "easy". For more on easiness, we refer to [11], [17].

2. Cube slicing. We are interested in the classification of the intermediate subgroups $H_{N} \subset G \subset U_{N}^{+}$. Such a quantum group can be imagined as sitting inside the cube, and the point is that by using the operations $\cap$ and $\langle$,$\rangle , we can "project" it on the faces and edges.$

In order to clarify this construction, let us start with the following definition:

Definition 2.1. Associated to any quantum group $H_{N} \subset G \subset U_{N}^{+}$are:

- its classical version, $G_{\text {class }}=G \cap U_{N}$;

- its free version, $G_{\text {free }}=\left\langle G, H_{N}^{+}\right\rangle$;

- its discrete version, $G_{\text {disc }}=G \cap K_{N}^{+}$;

- its continuous version, $G_{\text {cont }}=\left\langle G, O_{N}\right\rangle$;

- its real version, $G_{\text {real }}=G \cap O_{N}^{+}$;

- its unitary version, $G_{\text {unit }}=\left\langle G, K_{N}\right\rangle$.

Here we have chosen the word "unitary" instead of "complex" in order for the corresponding abbreviation "unit" not to be confused with "cont".

In connection with our cube, we can now formulate:

Proposition 2.2. Given an intermediate quantum group $H_{N} \subset G \subset U_{N}^{+}$, we have a diagram of closed subgroups of $U_{N}^{+}$, obtained by inserting
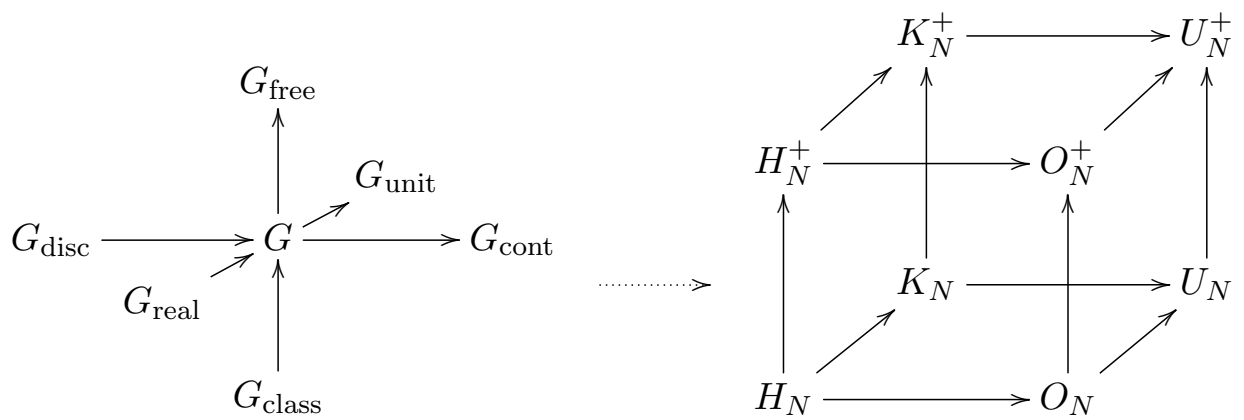

in the obvious way, with each $G_{x}$ belonging to the main diagonal of each face. 
Proof. The fact that we have indeed the diagram of inclusions on the left is clear from Definition 2.1 above. Regarding now the insertion procedure, consider any of the faces of the cube, $P \subset Q, R \subset S$. Our claim is that the corresponding quantum group $G_{x}$ can be inserted on the corresponding main diagonal $P \subset S$ :

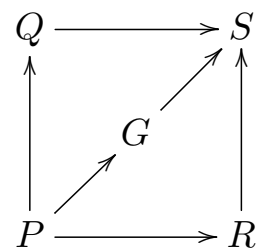

Thus, in order to finish, we have to check a total of $6 \times 2=12$ inclusions. But, according to Definition 2.1 above, the inclusions to be checked are:

(1) $H_{N} \subset G_{\text {class }} \subset U_{N}$, where $G_{\text {class }}=G \cap U_{N}$;

(2) $H_{N}^{+} \subset G_{\text {free }} \subset U_{N}^{+}$, where $G_{\text {free }}=\left\langle G, H_{N}^{+}\right\rangle$;

(3) $H_{N} \subset G_{\text {disc }} \subset K_{N}^{+}$, where $G_{\text {disc }}=G \cap K_{N}^{+}$;

(4) $O_{N} \subset G_{\text {cont }} \subset U_{N}^{+}$, where $G_{\text {cont }}=\left\langle G, O_{N}\right\rangle$;

(5) $H_{N} \subset G_{\text {real }} \subset O_{N}^{+}$, where $G_{\text {real }}=G \cap O_{N}^{+}$;

(6) $K_{N} \subset G_{\text {unit }} \subset U_{N}^{+}$, where $G_{\text {unit }}=\left\langle G, K_{N}\right\rangle$.

Since all these statements are trivial from the definition of $\cap$ and $\langle$,$\rangle , and$ from our assumption $H_{N} \subset G \subset U_{N}^{+}$, our insertion procedure works indeed, and we are done.

In order now to complete the diagram, we also have to project $G$ on the edges of the cube. For this purpose, we can assume that $G$ lies on one of the six faces.

The general result that we will need is as follows:

Proposition 2.3. Given an intersection/generation diagram $P \subset Q$, $R \subset S$ and an intermediate quantum group $P \subset G \subset S$, we have the following diagram:

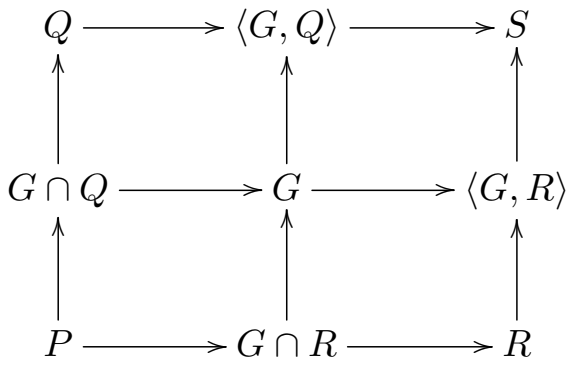

In addition, $G$ slices the square, in the sense that this is an intersection and generation diagram precisely when $G=\langle G \cap Q, G \cap R\rangle$ and $G=\langle G, Q\rangle \cap$ $\langle G, R\rangle$. 
Proof. This is indeed clear from the definitions, because the intersection and generation diagram conditions are automatic for the upper left and lower right squares, as also are half of the generation diagram conditions for the lower left and upper right squares.

Let us also record the Tannakian version of this construction:

Proposition 2.4. Given an intersection/generation diagram $P \subset Q$, $R \subset S$ and an intermediate quantum group $P \subset G \subset S$, we have the following diagram:

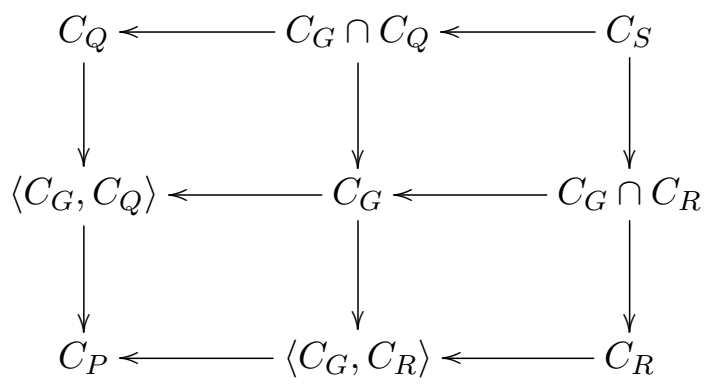

Also, $C_{G}$ slices the square, in the sense that this is an intersection and generation diagram precisely when $C_{P}=\left\langle C_{G}, C_{Q}\right\rangle \cap\left\langle C_{G}, C_{R}\right\rangle$ and $C_{S}=$ $\left\langle C_{G} \cap C_{Q}, C_{G} \cap C_{R}\right\rangle$.

Proof. This is indeed clear from the definitions, by proceeding as in the proof of Proposition 2.3. Observe that this is the diagram of Tannakian categories for the quantum groups in Proposition 2.3, due to the conversion formulae from Proposition 1.4.

Now back to the cube, given an intermediate subgroup $H_{N} \subset G \subset U_{N}^{+}$ we can perform the construction in Proposition 2.3 for each of the six faces of the cube. The problem, however, is that we will not obtain a diagram of inclusions in this way, because for each of the 12 edges of the cube, the corresponding "midpoint" will be defined twice.

As a first observation, six of these midpoints are actually well-defined, and there is no problem with them, because we have some compatibility formulae:

Proposition 2.5. We have the following results:

(1) $\left(G_{\text {class }}\right)_{\text {disc }}=\left(G_{\text {disc }}\right)_{\text {class }}=G \cap K_{N}$.

(2) $\left(G_{\text {class }}\right)_{\text {real }}=\left(G_{\text {real }}\right)_{\text {class }}=G \cap O_{N}$.

(3) $\left(G_{\text {disc }}\right)_{\text {real }}=\left(G_{\text {real }}\right)_{\text {disc }}=G \cap H_{N}^{+}$.

(4) $\left(G_{\text {free }}\right)_{\text {cont }}=\left(G_{\text {cont }}\right)_{\text {free }}=\left\langle G, O_{N}^{+}\right\rangle$.

(5) $\left(G_{\text {free }}\right)_{\text {unit }}=\left(G_{\text {unit }}\right)_{\text {free }}=\left\langle G, K_{N}^{+}\right\rangle$.

(6) $\left(G_{\text {cont }}\right)_{\text {unit }}=\left(G_{\text {unit }}\right)_{\text {cont }}=\left\langle G, U_{N}\right\rangle$. 
Proof. The formulae $(1,2,3)$ are all of the following type:

$$
(G \cap Q) \cap R=(G \cap R) \cap Q=G \cap P .
$$

As for $(4,5,6)$, these are all of the following type:

$$
\langle\langle G, Q\rangle, R\rangle=\langle\langle G, R\rangle, Q\rangle=\langle G, S\rangle .
$$

Thus the value of $G$ is in fact irrelevant, and the results simply follow from the fact that the cube is an intersection and generation diagram.

Regarding now the remaining six edges, the compatibility conditions here for the midpoints are not automatic, and we have to introduce the following notion:

Definition 2.6. We say that $G$ pre-slices the cube if it satisfies the following conditions:

- $\left(G_{\text {class }}\right)_{\text {cont }}=\left(G_{\text {cont }}\right)_{\text {class }}$.

- $\left(G_{\text {class }}\right)_{\text {unit }}=\left(G_{\text {unit }}\right)_{\text {class }}$.

- $\left(G_{\text {disc }}\right)_{\text {free }}=\left(G_{\text {free }}\right)_{\text {disc }}$.

- $\left(G_{\text {disc }}\right)_{\text {unit }}=\left(G_{\text {unit }}\right)_{\text {disc }}$.

- $\left(G_{\text {real }}\right)_{\text {free }}=\left(G_{\text {free }}\right)_{\text {real }}$.

- $\left(G_{\text {real }}\right)_{\text {cont }}=\left(G_{\text {cont }}\right)_{\text {real }}$.

In other words, we are asking here for each intersection operation in Definition 2.1 to commute with the generation operations, except for the "opposite" operation.

We can now formulate our first slicing result:

Proposition 2.7. Assuming that $G$ pre-slices the cube in the above sense, the diagram in Proposition 2.2 can be completed, via the construction in Proposition 2.3, to a diagram fully slicing the cube along the three coordinate axes, into eight small cubes.

Proof. As already mentioned, checking that the conclusion holds is a matter of checking that the 12 projections on the edges are well-defined. And the situation is as follows:

(1) Regarding the three edges emanating from $H_{N}$, and the three edges landing in $U_{N}^{+}$, the result follows from the formulae in Proposition 2.5.

(2) For the remaining six edges, not emanating from $H_{N}$ or landing in $U_{N}^{+}$, the result follows from the formulae in Definition 2.6.

We are not done with the slicing work yet, because nothing guarantees that our slicing is "neat", in the sense that we obtain an intersection and generation diagram. In order to have this property, we have to introduce one more definition:

Definition 2.8. We say that $G$ slices the cube when

- $G$ pre-slices the cube in the sense of Definition 2.6, 
- $G_{\text {class }}, G, G_{\text {free }}$ slice the classical/intermediate/free faces,

- $G_{\text {disc }}, G, G_{\text {cont }}$ slice the discrete/intermediate/continuous faces,

- $G_{\text {real }}, G, G_{\text {unit }}$ slice the real/intermediate/unitary faces,

where by "intermediate" we mean in each case "parallel to its neighbors".

In short, we are asking here for a total of $6 \times 4=24$ conditions to be satisfied, namely the six slicing conditions from Definition 2.6, and then the $6 \times 3=18$ conditions coming from the two conditions in Proposition 2.3, applied to the $3 \times 3$ faces of the slicing.

We can now finish the slicing procedure:

TheOrem 2.9. Assuming that $G$ slices the cube in the above sense, we have a diagram fully slicing the cube into eight small cubes, which is an intersection diagram, in the sense that each of its 36 small square faces is an intersection and generation diagram.

Proof. Here the first assertion follows from Proposition 2.7, and the second from Proposition 2.3, via our assumptions from Definition 2.8.

Summarizing, we know now how to slice the cube, with the remark that the $36 \times 2=72$ intersection and generation properties that are a priori needed, for the various small faces, collapse in fact to the $6 \times 4=24$ conditions from Definition 2.8.

Of course, this $72 \rightarrow 24$ simplification is trivial, simply coming from the fact that the cube is an intersection and generation diagram, and not involving $G$ itself. It is probably possible to do slightly better here by careful inspection.

However, when going on this way, at some point there will certainly be a need for a deep result. So, the problem of fully simplifying our axioms is open, and interesting.

3. Classification. All the above is quite natural, and looking for quantum groups having the slicing property is an interesting question. However, our purpose here is a bit different: we are interested in formulating a foundational result, rather than something technical.

In order to do so, we introduce one more concept:

Definition 3.1. A family of compact quantum groups $G=\left(G_{N}\right)$, with $G_{N} \subset U_{N}^{+}$for any $N \in \mathbb{N}$, is called uniform when

$$
G_{N-1}=G_{N} \cap U_{N-1}^{+}
$$

with respect to the standard embeddings $U_{N-1}^{+} \subset U_{N}^{+}$, given by $u=\operatorname{diag}(1, v)$.

This condition is very natural, algebrically speaking, because we are here in an injective/projective limit situation for the associated compact and discrete quantum groups. At a more advanced level, this condition appeared 
in [11], in connection with the Bercovici-Pata bijection [13] for the asymptotic laws of truncated characters, and also in [1], [10], in connection with various noncommutative geometry questions. See [3].

We can now prove the statement announced in the introduction, namely:

TheOrem 3.2. Assume that $H_{N} \subset G \subset U_{N}^{+}$has the following properties:

- Easiness.

- Uniformity.

- Slicing property.

Then $G$ must be one of the basic eight quantum groups.

Proof. The idea will be that of "locating" our quantum group inside the cube, in a 3D sense, by using the slicing property. There are many ways of doing so, and in view of the known classification results, whose technical level can vary a lot, the best is by using the results for the classical face from [26], and the results for the orthogonal edge from [12]. In other words, we would like to use the "coordinate system" highlighted below:

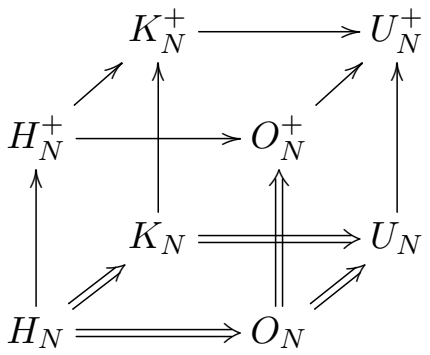

Let us start with the classical face. Our goal here is to find the possible values of $G_{\text {class }}$, which belong by definition to this face. In order to simplify the discussion, we will temporarily assume $G=G_{\text {class }}$. Thus, we would like to find the intermediate quantum groups $H_{N} \subset G \subset U_{N}$ which are easy, uniform, and which slice the lower face.

According to Proposition 2.3, the slicing diagram for the lower face is

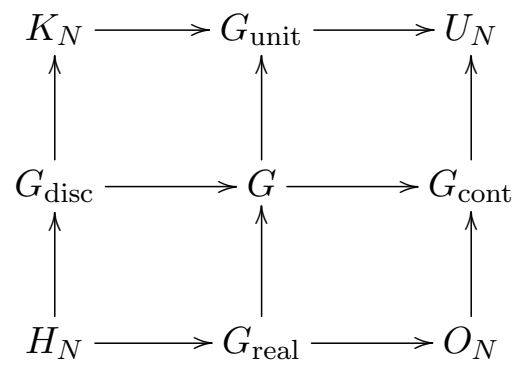

With these preliminaries in hand, we can now survey the known results on the subject. There are several statements here, all based on [26]: 
Classical face, easy case. The full classification of the intermediate easy quantum groups $H_{N} \subset G \subset U_{N}$ is available from [26]. There are many examples here, with the whole subject being quite technical, and we refer to [26] for the full details.

Classical face, easy uniform case. As explained in [1, in the context of the noncommutative homogeneous space considerations there, which require the uniformity axiom, imposing this axiom leads to some simplifications, the solutions being as follows:

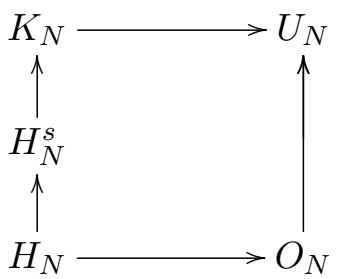

Here the extra groups on the left are the complex reflection groups $H_{N}^{s}=$ $\mathbb{Z}_{s} \prec S_{N}$ with $s \in\{2,4,6, \ldots, \infty\}$ from [5], which at $s=2, \infty$ cover $H_{N}, K_{N}$. See [1].

Classical face, easy slicing case. As explained in [3], when imposing the slicing condition on the lower face, which comes from the general noncommutative geometry considerations in [2, [6], some simplifications appear as well, the solutions being as follows:

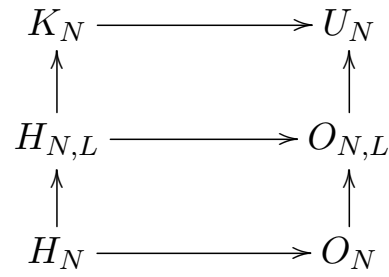

Here the various extra groups are obtained by "arithmetic complexification", according to the formula $G_{L}=\mathbb{Z}_{L} G$, with $L \in\{2,3, \ldots, \infty\}$. See [2], [3], [6].

Classical face, easy uniform slicing case. In order to obtain the solutions here, we just have to intersect the above two diagrams, and we obtain

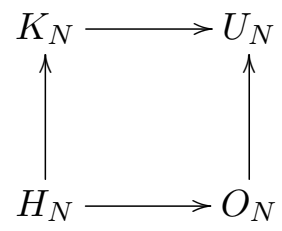

In short, getting back to our original problem, we have reached the conclusion that $G_{\text {class }}$ must be one of the four vertices of the lower face of the cube. 
With this result in hand, we can now go ahead, and finish by using [12]. Indeed, the projection of $G$ on the real continuous edge, $O_{N} \subset O_{N}^{+}$, must be an intermediate easy quantum group $O_{N} \subset G \subset O_{N}^{+}$. But, according to [12], the only nontrivial solution here is the half-classical orthogonal group $O_{N}^{*}$, coming from the half-commutation relations $a b c=c b a$. And since this quantum group is not uniform, simply because $a b c=c b a$ with $c=1$ imply $a b=b a$, as explained in [10, we have only $O_{N}, O_{N}^{+}$as solutions.

Summarizing, our intermediate quantum group $H_{N} \subset G \subset U_{N}^{+}$must lie on the upper or the lower face of the cube, and its projection on the lower face must be one of the four vertices of the lower face. Thus $G$ must be one of the eight vertices of the cube, as claimed.

4. Conclusion. We have seen that by "piling up" a number of axioms, which are natural in the noncommutative geometry and free probability context, and which actually came from a substantial amount of work in this direction, we are left with eight quantum groups.

All this is of course quite philosophical. What we have here is rather some kind of "ground zero" result, providing a foundational framework for more specialized classification results, which can be obtained by carefully modifying or removing the axioms.

Here is a brief discussion, regarding the modification/removal of these axioms:

Problem 4.1. Modify or remove the slicing axiom.

Generally speaking, totally removing the slicing axiom leads to some difficult questions, with the problem coming from lack of 3D orientation inside the cube.

One fruitful direction, however, comes by restricting attention to the six faces of the cube, and trying to find uniform easy quantum groups which slice the face.

Skipping the details here, let us mention that the problem is solved in [26] for the upper and lower faces; it is elementary as well for the front and right face, using the results from [12], [26], and is still in need of some non-trivial combinatorial work, based on the results in [23], 24], 25], as regards the left face and the bottom face.

Problem 4.2. Modify or remove the uniformity axiom.

This is another interesting direction. The general strategy from the proof of Theorem 3.2 above can be followed, with the only piece of work still needed being to unify the constructions on $H_{N, L}, O_{N, L}$ with the construction of the half-liberations.

Problem 4.3. Modify or remove the easiness axiom. 
This is something heavier. In principle the general strategy from the proof of Theorem 3.2 above can be followed too, the work in the classical case being probably quite standard, and with conjectural input for the orthogonal edge coming from [8].

There are also several modifications of the easy quantum group theory which can be used, the most standard ones, at least for now, coming from the work in [9], [19], [20].

Problem 4.4. Modify or remove the $H_{N} \subset G$ axiom.

Once again, this is heavier. A natural direction, which would however require rethinking the slicing procedure, is that of using the condition $S_{N} \subset G$. Note that this would also require dealing with all the "singleton issues" which might appear.

Along the same lines, using some even weaker conditions, of type $A_{N} \subset G$, makes sense as well, at least theoretically. For some comments here, we refer to 4 .

\section{Problem 4.5. Modify everything.}

This is somewhat philosophical. Let us remember that the extra axioms in Theorem 3.2 were obtained by "putting to work" compact quantum groups, in connection with various questions in noncommutative geometry and free probability. There are of course many other potential applications of compact quantum groups, and putting them to work on other topics, with some axiomatics in mind, could perfectly lead, in the long run, to a different philosophy, different axioms, and a different "ground zero" result.

Having such an alternative work done would be of course immensely useful.

\section{References}

[1] T. Banica, Liberation theory for noncommutative homogeneous spaces, Ann. Fac. Sci. Toulouse Math. 26 (2017), 127-156.

[2] T. Banica, Unitary easy quantum groups: geometric aspects, J. Geom. Phys. 126 (2018), 127-147.

[3] T. Banica, Quantum groups, from a functional analysis perspective, Adv. Oper. Theory 4 (2019), 164-196.

[4] T. Banica, Homogeneous quantum groups and their easiness level, Kyoto J. Math., to appear.

[5] T. Banica, S. T. Belinschi, M. Capitaine and B. Collins, Free Bessel laws, Canad. J. Math. 63 (2011), 3-37.

[6] T. Banica and J. Bichon, Complex analogues of the half-classical geometry, Münster J. Math. 10 (2017), 457-483.

[7] T. Banica, J. Bichon and B. Collins, The hyperoctahedral quantum group, J. Ramanujan Math. Soc. 22 (2007), 345-384.

[8] T. Banica, J. Bichon, B. Collins and S. Curran, A maximality result for orthogonal quantum groups, Comm. Algebra 41 (2013), 656-665. 
[9] T. Banica and A. Skalski, Two-parameter families of quantum symmetry groups, J. Funct. Anal. 260 (2011), 3252-3282.

[10] T. Banica, A. Skalski and P. M. Sołtan, Noncommutative homogeneous spaces: the matrix case, J. Geom. Phys. 62 (2012), 1451-1466.

[11] T. Banica and R. Speicher, Liberation of orthogonal Lie groups, Adv. Math. 222 (2009), 1461-1501.

[12] T. Banica and R. Vergnioux, Invariants of the half-liberated orthogonal group, Ann. Inst. Fourier (Grenoble) 60 (2010), 2137-2164.

[13] H. Bercovici and V. Pata, Stable laws and domains of attraction in free probability theory, Ann. of Math. 149 (1999), 1023-1060.

[14] J. Bichon, Free wreath product by the quantum permutation group, Algebras Represent. Theory 7 (2004), 343-362.

[15] M. Brannan, A. Chirvasitu and A. Freslon, Topological generation and matrix models for quantum reflection groups, preprint, 2018.

[16] R. Brauer, On algebras which are connected with the semisimple continuous groups, Ann. of Math. 38 (1937), 857-872.

[17] B. Collins and P. Śniady, Integration with respect to the Haar measure on unitary, orthogonal and symplectic groups, Comm. Math. Phys. 264 (2006), 773-795.

[18] V. G. Drinfeld, Quantum groups, in: Proc. ICM (Berkeley, 1986), 798-820.

[19] A. Freslon, On the partition approach to Schur-Weyl duality and free quantum groups, Transform. Groups 22 (2017), 707-751.

[20] A. Freslon, On two-coloured noncrossing quantum groups, preprint, 2017.

[21] M. Jimbo, A q-difference analog of $U(\mathfrak{g})$ and the Yang-Baxter equation, Lett. Math. Phys. 10 (1985), 63-69.

[22] S. Malacarne, Woronowicz's Tannaka-Krein duality and free orthogonal quantum groups, Math. Scand. 122 (2018), 151-160.

[23] A. Mang and M. Weber, Categories of two-colored pair partitions, part I: Categories indexed by cyclic groups, preprint, 2018.

[24] A. Mang and M. Weber, Categories of two-colored pair partitions, part II: Categories indexed by semigroups, preprint, 2019.

[25] S. Raum and M. Weber, The full classification of orthogonal easy quantum groups, Comm. Math. Phys. 341 (2016), 751-779.

[26] P. Tarrago and M. Weber, Unitary easy quantum groups: the free case and the group case, Int. Math. Res. Notices 2017, 5710-5750.

[27] S. Wang, Free products of compact quantum groups, Comm. Math. Phys. 167 (1995), 671-692.

[28] S. Wang, Quantum symmetry groups of finite spaces, Comm. Math. Phys. 195 (1998), 195-211.

[29] S. L. Woronowicz, Compact matrix pseudogroups, Comm. Math. Phys. 111 (1987), 613-665.

[30] S. L. Woronowicz, Tannaka-Krein duality for compact matrix pseudogroups. Twisted $S U(N)$ groups, Invent. Math. 93 (1988), 35-76.

Teo Banica

Department of Mathematics

University of Cergy-Pontoise

F-95000 Cergy-Pontoise, France

E-mail: teo.banica@gmail.com 
